\title{
Fallbeispiel einer lebensbedrohlichen Komplikation in der Geburtshilfe
}

\author{
Prof. Dr. Werner Rath, Kiel
}

\section{Die Fruchtwasserembolie (FWE) ist mit einer Inzidenz von 2-8/100.000 Geburten zwar eine seltene, aber für Mutter und Kind immer lebensbedrohliche Komplikation: Die mütterliche Sterblichkeit liegt bei 13,5 bis $70 \%$, die perinatale Mortalität bei 7 bis $38 \%$. Insbesondere bei einer antenatalen FWE (55 bis $76 \%$ der Fälle) ist die Hebamme häufig die erste, die mit den Symptomen einer FWE konfrontiert wird. Ihr kommt daher eine Schlüsselrolle vor allem in der raschen, unter Umständen lebensrettenden Diagnose-} stellung zu.

\section{Falldarstellung}

Frau J. freute sich mit ihren 39 Jahren auf ihr drittes Kind. Vorausgegangen waren zwei unkomplizierte Spontangeburten. Aus ihrer Vorgeschichte waren Asthma bronchiale sowie diverse Allergien (z.B. Hausstaub, Penicillin) bekannt. Frau J. hatte in der 38. SSW eine milde Präeklampsie entwickelt und wurde daher zur Geburtseinleitung in die Klinik eingewiesen.

Hebamme Frau M. und Assistenzarzt Dr. L. (zurzeit im 3. Weiterbildungsjahr) erwarteten sie schon im Kreißsaal. Die Aufnahmeuntersuchung ergab ein unauffälliges CTG, Blutdruck 145/95 mmHg, Teststreifen: Eiweiß ++, Zervix $2 \mathrm{~cm}$ lang, Muttermund Fingerkuppe einlegbar, Ultraschall: Junge in Schädellage, normale Fruchtwassermenge, Plazenta unauffällig.

Nach dem Aufklärungsgespräch über die Geburtseinleitung entschied man sich für eine vaginale Applikation von Prostaglandin-E2-Gel. „Die Einleitung dürfte keine Probleme machen, Sie haben ja bereits zwei Kinder auf natürlichem Wege zur Welt gebracht“, beruhigte Dr. L. Frau J. Es war 10.00 Uhr, als das erste Prostaglandin-Gel eingelegt wurde.
Frau J. hatte keine Angst vor der Geburtseinleitung, sie freute sich jetzt auf ihren kleinen Sohn, der Carlo heißen sollte. Alles lief wie geplant: Zwei Stunden nach der Prostaglandin-Gabe bekam Frau J. zunehmend Wehen, die sie gut veratmen konnte. Dann ließen bei einer Muttermundsweite von 2-3 cm die Wehen wieder nach. Das geburtshilfliche Team entschied sich, Oxytocin je nach Wehentätigkeit in ansteigender Dosierung intravenös zu geben. Gegen 15.00 Uhr kam es bei einer Muttermundsweite von 4-5 cm und fest eingestelltem kindlichen Köpfchen und bis dahin unauffälligem CTG zum spontanen Blasensprung... Und dann passierte es sozusagen „aus heiterem Himmel“.

Gerade hatte sich Frau J. noch mit Hebamme Frau M. über mögliche Namen des Sohnes unterhalten, da durchfuhr sie plötzlich ein Kältegefühl und ein Kribbeln im ganzen Körper.

Hinzu kamen „Lichtblitze“ vor den Augen, sie spürte eine große Unruhe und ein zunehmendes Druckgefühl in der Brust. Am schlimmsten aber waren die jetzt einsetzende Kurzatmigkeit und Atemnot. Dann konnte Frau J. die Stimme von Hebamme Frau M. nicht mehr hören. „Frau J., können Sie mich verstehen?“, rief Frau M. und rüttelte an Frau J.s Schultern. Sie bemerkte trotz ihrer Aufregung sofort, dass etwas nicht stimmte. In diesem Augenblick steckte ihre Kollegin Hebamme Frau S. den Kopf durch die Tür des Entbindungszimmers. „Benachrichtige sofort Dr. L. und Oberarzt Dr. K., auch am besten gleich den diensthabenden Oberarzt der Anästhesie. Die Patientin ist bewusstlos!“, fuhr es aus Hebamme Frau M. heraus.

Die Klinik verfügte für geburtshilfliche Notfälle sowohl über interdisziplinär abgestimmte Handlungsanweisungen als auch über einen logistischen Notfallplan: „Wann, wen, unter welcher Telefonnummer benachrichtigen?" Das hatten sie wiederholt im Team in Echtzeit trainiert.

Das „Ereignis“ trat um 15.10 Uhr ein, um 15.15 Uhr waren alle vor Ort. Hebamme Frau M. hatte regelmäßig Blutdruck und Puls gemessen, auch - eher zufällig - unmittelbar vor Frau J.s Bewusstlosigkeit: Blutdruckabfall innerhalb von 1-2 Minuten von 140/90 mmHg auf 70-50 mmHg, Pulsanstieg von 90/min. auf 130/min. Oberarzt A. aus der Anästhesie nahm diese Werte zur Kenntnis. Der Anästhesiepfleger klebte in aller Eile EKG-Elektroden auf. 
Der Kreislaufmonitor zeigte zunächst eine kardiale Arrhythmie und dann die Katastrophe - akuter Herz-Kreislaufstillstand!

Frau J. war inzwischen zyanotisch geworden. Die Pulsoxymetrie ergab eine Sauerstoffsättigung von knapp $80 \%$. Nun musste alles ganz schnell gehen: endotrachiale Intubation, invasive Beatmung und externe Herzmassage, Volumengabe mit kristalloiden Lösungen und intravenöse Applikation von Katecholaminen (siehe Abb.1). Glücklicherweise kam es infolge dieser Maßnahmen zu einer raschen Wiederherstellung der Herz-Kreislauffunktionen und zu einer adäquaten Oxygenierung. Währenddessen fragte Oberarzt K., an Hebamme Frau M. gewandt: „Was ist denn mit dem Kind?“ In der ersten Aufregung hatte man eine bereits kurz nach dem Ereignis aufgetretene prolongierte Dezeleration von unter 80 Schlägen/min. im CTG übersehen. Gerade war eine weitere Dezeleration gefolgt.

„Wir müssen sofort die Not-Sectio durchführen, das ist auch besser für das Kind“, rief Oberarzt $K$.
Da das hierfür notwendige Team bereits an Ort und Stelle war, konnte die NotSectio im Kreißsaal-OP unverzüglich vorgenommen werden. Sie verlief ohne Probleme. Der kleine Carlo kam in etwas deprimiertem Zustand zur Welt und wurde von dem inzwischen anwesenden Neonatologen versorgt (Apgar 5/8/9; Nabelschnur-pH: 7,18). Der Blutverlust betrug $700 \mathrm{ml}$, am Ende der Operation war der Uterus gut tonisiert, Fundusstand ein Querfinger oberhalb des Nabels.

Frau J. wurde intubiert auf die Anästhesie-Wachstation verlegt (17.10 Uhr). Dort wurde gleich nach der Aufnahme ein zweites Notfalllabor abgenommen (das erste Notfalllabor erfolgte zu Beginn der Sectio). Unter Dobutamin war Frau J.s Herz-Kreislauf-Funktion stabil bei jetzt ausreichender Sauerstoffsättigung unter Beatmung.

Als Anästhesieschwester Frau C. gegen 18.00 Uhr die Vorlage wechseln wollte, bekam sie einen „Riesenschreck“: Die Vorlage war komplett durchgeblutet, weiteres wässriges Blut in den Bettlaken, Blut zwischen den Beinen der Patientin,

\section{Fruchtwasserembolie: Pathogenese — Symptome - Behandlung}

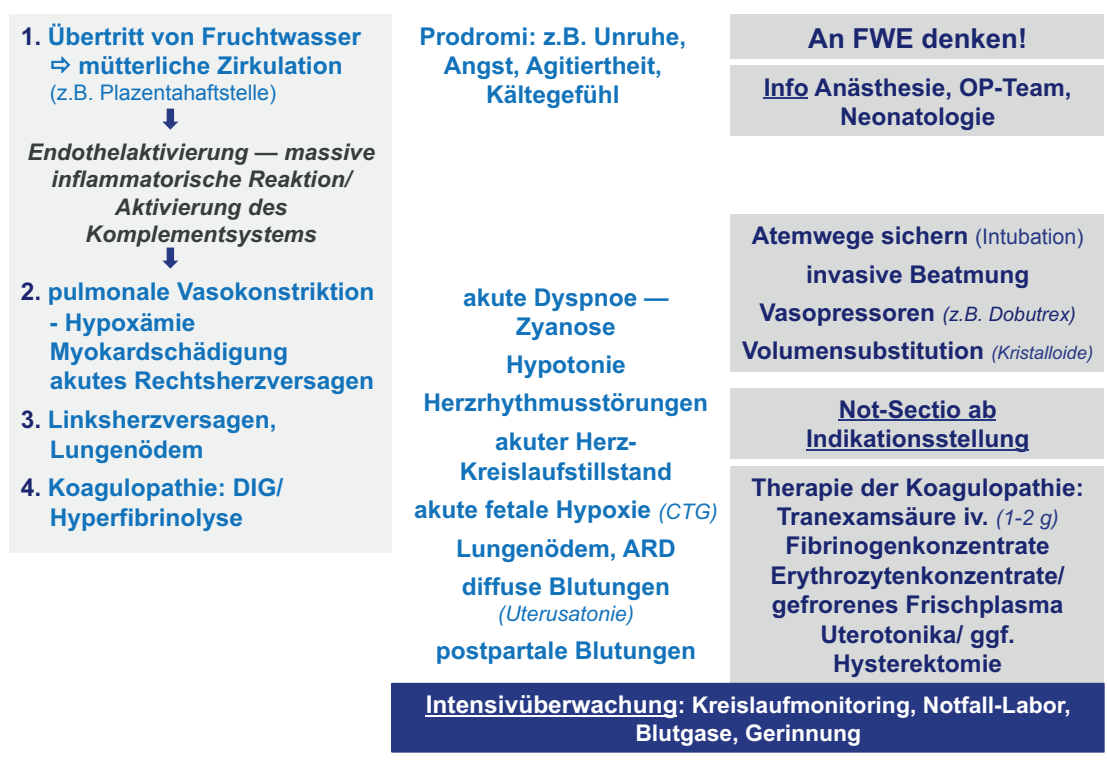

Abb. 1 Hebammen sollten die Symptome einer Fruchtwasserembolie kennen. Zudem ist ein logistischer Ablaufplan für Notfälle im Kreißsaal unerlässlich. außerdem war die Kompresse auf der Laparotomiewunde durchgeblutet. Wenig später war das geburtshilfliche Team, welches sofort benachrichtigt wurde, bei Frau J.

Oberarzt K. verschaffte sich kurz einen Überblick über die Situation: starke vaginale Blutung, keine Koagelbildung, Blut gerinnt nicht mehr richtig, Uterus völlig schlaff.

Inzwischen waren die zuletzt abgenommenen Laborwerte eingetroffen, die der Oberarzt jetzt mit denen von 15.20 Uhr verglich: Hämoglobin: 11,8 g/dl $\rightarrow$ 7,2 g/dl, Thrombozyten: 154 G/l $\rightarrow 35$ G/l, Quick: $80 \% \rightarrow 20 \%$, Fibrinogen: $4,8 \mathrm{mg} / \mathrm{dl} \rightarrow$ $0,5 \mathrm{mg} / \mathrm{dl}$.

„Wir brauchen sofort Oxytocin, am besten 40 I.E. in 500 ml Ringerlactat, wahrscheinlich auch Sulproston. Außerdem müssen wir dringend etwas für die Gerinnung tun!“, rief Dr. K. Schwester Frau C. und dem anwesenden Anästhesisten Dr. F. zu. Während sich das Anästhesieteam um die Korrektur der Gerinnungsstörung kümmerte (initial $1 \mathrm{~g}$ Tranexamsäure intravenös, $4 \mathrm{~g}$ Fibrinogenkonzentrat $\rightarrow$ Erythrozytenkonzentrate und gefrorenes Frischplasma im Verhältnis 1:1, Thrombozytenkonzentrate), musste Oberarzt K. feststellen, dass der Uterus zunächst auf Oxytocin und dann auch auf Sulproston in ansteigender Dosierung nicht reagierte. Der Uterus hatte immer wieder schlaffe, atone Phasen, es blutete weiter, wenn auch nicht mehr so stark wie vorher. „Wenn es so weiter blutet, kann ich die Herz-Kreislauffunktion trotz aller Maßnahmen nicht mehr lange stabil halten“, sagte Oberarzt F. besorgt.

Es blieb in dieser erneut lebensbedrohlichen Situation nur die Hysterektomie, die dann ca. 30 Minuten später durchgeführt wurde.

Frau J. überstand trotz nochmaligen Blutverlustes von ca. 1,5 Litern die Operation unter massiver Substitution von Erythrozytenkonzentraten, gefrorenem Frischplasma, Fibrinogen- und Thrombo- 
zytenkonzentraten ohne Komplikationen. Dabei kam ihr zugute, dass sie außer dem Asthma eine gesunde Frau war. 16 Stunden nach der Operation konnte Frau J. extubiert werden, weitere Blutungen traten nicht auf, auch keine neurologischen Folgestörungen. Am 14. Tag nach Aufnahme war es dann soweit: Mutter und Kind konnten entlassen werden.

Frau J. brauchte noch Monate, um dieses „Trauma“ zu verarbeiten. Eine Psychologin unterstützte sie dabei.

\section{Kommentar}

Mütterliches Alter > 35 Jahre, allergische Diathesen, milde Präeklampsie und die Geburtseinleitung mit Wehenverstärkung durch Oxytocin (wie bei Frau J.) gelten als Risikofaktoren für eine Fruchtwasserembolie. Stärkere Risikofaktoren sind: Sectio caesarea, Plazenta praevia und Mehrlingsschwangerschaft [1]. Die FWE tritt entweder aus völliger Gesundheit oder - wie im vorliegenden Fall nach Prodromalsymptomen wie Kältegefühl, Kribbeln, Unruhe und Angst auf.

Nach internationalen Diagnosekriterien sollte bei akuter kardiovaskulärer Dekompensation ohne andere klar erkennbare Ursachen an eine FWE gedacht werden (Ausschlussdiagnose), wenn eine oder mehrere der folgenden Symptome vorliegen [3]:

- akut einsetzende Dyspnoe

- Zyanose

- Hypotension

- Herzrhythmusstörungen

- plötzlicher Herz-Kreislaufstillstand

- akute fetale Hypoxie (CTG)

- neurologische Symptome, z.B. Krämpfe

Die Diagnose beweisende laborchemische und apparative Verfahren stehen bisher für die klinische Praxis nicht zur Verfügung [2].

Für Frau J. möglicherweise lebensrettend war, dass Hebamme Frau M. den Notfall rechtzeitig erkannt und sofort ärztliche Hilfe angefordert hat.
Die Hebamme sollte daher die Symptome der FWE kennen, sie sollte sofern kein Arzt zur Stelle ist - auch nicht zögern, mit Erstmaßnahmen zur Reanimation zu beginnen [4]:

\section{- A: Atmung sichern}

- B: Beatmung, notfalls auch Mund-zuMund, Sauerstoffgabe

- C: Kreislaufüberwachung, Infusion mit kristalloiden Lösungen anhängen

Für die Praxis bedeutet das: Nicht nur Ärzte, sondern auch Hebammen sollten regelmäßig an Schulungs-/Trainingsprogrammen zur Reanimation des Erwachsenen (und des Neugeborenen) teilnehmen.

Besonders wichtig - wie der vorliegende Fall zeigt - sind für geburtshilfliche Notfälle im Kreißsaal verfügbare, interdisziplinär abgestimmte und Leitlinien-orientierte Handlungsanweisungen/-algorithmen sowie ein logistischer Notfallplan („Roter Knopf“ im Kreißsaal, strukturierte Benachrichtigungskette) mit klaren Aufgabenverteilungen. Diese Abläufe müssen in regelmäßigen Abständen in Echtzeit trainiert werden. Ausweislich aktueller internationaler Publikationen tragen diese Maßnahmen signifikant zu einer Verminderung der mütterlichen Morbidität und Mortalität bei.

Häufigste Differenzialdiagnosen der FWE sind die akute Lungenembolie (typische Risikofaktoren, meist postpartal, klinisches Leitsymptom: Thoraxschmerz, fehlt bei der FWE) und der akute Myokardinfarkt (typische Risikofaktoren in der Anamnese, Leitsymptome: substernale Schmerzen mit Ausstrahlung, kardiale Arrhythmien) [5].

Nach Anwesenheit des Geburtshelfers/ Anästhesisten im Kreißsaal kommen der Hebamme wichtige Aufgaben innerhalb der Teamarbeit zu, u. a. [6]:

- rasche Bereitstellung von Infusionslösungen, Medikamenten

- Benachrichtigung der Blutbank, des Notfalllabors und der Kinderärzte
- Beschaffung von Blutprodukten

- Vorbereitung der Not-Sectio

- postpartale Überwachung, z. B. Blutverlust beurteilen, Uterustonus und Fundusstand prüfen.

Frau J. profitierte nicht nur von der frühen Diagnosestellung und dem raschen Handeln der Hebamme, sondern auch von einer gut funktionierenden Infrastruktur der Klinik. Jede/jeder geburtshilflich Tätige weiß, dass diese optimalen Voraussetzungen und Bedingungen (wie im vorliegenden Fall) nicht zu jeder Zeit in jeder geburtshilflichen Abteilung zu gewährleisten sind. Dies trifft auch für die Not-Sectio zu, die bei mütterlichem Herzstillstand möglichst innerhalb von 5 Minuten erfolgen sollte. Sie erhöht die Chancen des Neugeborenen auf ein Überleben ohne neurologische Störungen und verbessert durch die Entleerung des Uterus den venösen Rückstrom über die Vena cava inferior zum Herzen der Mutter [7].

Was im vorliegenden Fall möglicherweise nicht adäquat berücksichtigt wurde, ist die Entwicklung einer Koagulopathie, verursacht durch eine massive Hyperfibrinolyse mit Hypofibrinogenämie oder durch die Entwicklung einer disseminierten intravasalen Gerinnung mit Verbrauchskoagulopathie. Nach Überleben der Initialphase der FWE muss in 30$45 \%$ der Fälle mit einer manifesten Gerinnungsstörung in einem Zeitraum zwischen 10-30 Minuten (50\% innerhalb von $4 \mathrm{Std}$.) bis zu 9 Stunden nach Eintritt der FWE gerechnet werden [8]. Zudem ist das Risiko für eine schwere, therapierefraktäre Uterusatonie erhöht. An diese, mit unterschiedlicher Latenzzeit auftretenden Komplikationen der FWE muss unbedingt gedacht werden! Wahrscheinlich wäre die Latenzzeit zwischen Beginn bis zum Erkennen der Koagulopathie und Uterusatonie unter Überwachungskontrolle einer Hebamme/eines Geburtshelfers kürzer gewesen. Es ist daher wichtig, dass geburtshilfliche Notfälle mit Verlegung auf die AnästhesieWachstation geburtshilflich engmaschig mitbetreut werden. 


\section{Fazit}

Die optimale Zusammenarbeit von geschulten Hebammen, Geburtshelfern und Anästhesisten sowie das Verantwortungsbewusstsein der Krankenhausverwaltung/-träger der werdenden Familie gegenüber (ausreichende personelle, räumliche und apparative Infrastruktur) sind maßgebliche Voraussetzungen, dass schwere geburtshilfliche Notfälle wie die FWE nicht in einer Katastrophe, sondern im gesunden Überleben von Mutter und Kind enden.

\section{Literatur}

1 Benson MD. Amniotic fluid embolism: the known and not known. Obstet Med 2014; 7: 17-21

2 Rath WH, Hofer S, Sinicina J. Fruchtwasserembolie - eine interdisziplinäre Herausforderung: Epidemiologie, Diagnostik und Therapie. Dtsch Ärztebl 2014; 111 (8): 126-132
3 Knight M, Tufnell D, Brocklehurst P et al. on behalf on the UKOSS: Incidence and risk factors for amniotic fluid embolism. Obstet Gynecol 2010; 115: 910-917

4 Balinger KJ, Lam MTC, Hon HH et al. Amniotic fluid embolism: despite progress, challenges remain. Curr Opin Obstet Gynecol 2015; 27: 398-405

5 Rath W. Herzstillstand während und nach der Geburt: Fruchtwasserembolie Lungenembolie - Myokardinfarkt peripartale Kardiomyopathie. Gynpraktische Gynäkologie 2015; 20 : 191-200

6 Rath W. Fruchtwasserembolie - worst case. Deutsche Hebammenzeitschrift 2015; 4: 19-22

7 Gist RS, Stafford IP, Leibowitz AB, Ballin Y. Amniotic fluid embolism. Anesth Analg 2009; 108: 1599-1602

8 Bean LS, Rogers RP, Harley FA, Hood DD. Case Scenario: amniotic fluid embolism. Anesthesiology 2012; 116: 186-192

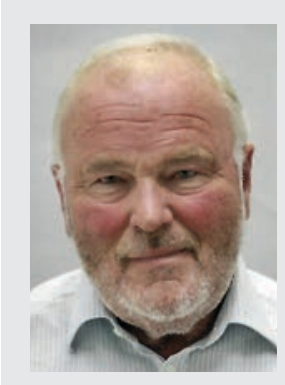

Univ.-Prof. Dr. Werner Rath Universitätsklinikum Schleswig-Holstein Arnold-Heller-Str. 3 24105 Kiel E-Mail: Werner.Rath@uksh.de 